American Journal of Applied Sciences 9 (2): 202-207, 2012

ISSN 1546-9239

(C) 2012 Science Publications

\title{
Complexity of Cocktail Party Graph and Crown Graph
}

\author{
Daoud, S.N. \\ ${ }^{1}$ Department of Applied Mathematics, Faculty of Applied Science, \\ Taibah University, Al-Madinah, K.S.A. \\ ${ }^{2}$ Department of Mathematics, Faculty of Science, \\ El-Minufiya University, Shebeen El-Kom, Egypt
}

\begin{abstract}
Problem statement: The number of spanning trees $\tau(\mathrm{G})$ in graphs (networks) was an important invariant. Approach: Using the properties of the Chebyshev polynomials of the second kind and the linear algebra techniques to evaluate the associated determinants. Results: The complexity, number of spanning trees, of the cocktail party graph on $2 \mathrm{n}$ vertices, given in detail in the text was proved. Also the complexity of the crown graph on $2 \mathrm{n}$ vertices was shown to had the value $\mathrm{n}^{\mathrm{n}-2}(\mathrm{n}-1)$ $(n-2)^{n-1}$. Conclusion: The number of spanning trees $\tau(G)$ in graphs (networks) is an important invariant. The evaluation of this number and analyzing its behavior is not only interesting from a mathematical (computational) perspective, but also, it is an important measure of reliability of a network and designing electrical circuits. Some computationally hard problems such as the travelling salesman problem can be solved approximately by using spanning trees. Due to the high dependence of the network design and reliability on the graph theory we introduced the above important theorems and lemmas and their proofs.
\end{abstract}

Key words: Complexity of graphs, spanning trees, cocktail party graphs, chebyshev polynomials, crown graph

\section{INTRODUCTION}

In this introduction we give some basic definitions and lemmas. We deal with simple and finite undirected graphs $\mathrm{G}=(\mathrm{V}, \mathrm{E})$, where $\mathrm{V}$ is the vertex set and $E$ is the edge set. For a graph $G$, a spanning tree in $G$ is a tree which has the same vertex set as $\mathrm{G}$. The number of spanning trees in $\mathrm{G}$, also called, the complexity of the graph, denoted by $\tau(\mathrm{G})$, is a well-studied quantity (for long time). A classical result of Kirchhoff (1847) can be used to determine the number of spanning trees for $\mathrm{G}=(\mathrm{V}, \mathrm{E})$. Let $\mathrm{V}=$ $\left\{\mathrm{v}_{1}, \mathrm{v}_{2}, \ldots, \mathrm{v}_{\mathrm{n}}\right\}$, then the Kirchhoff matrix $\mathrm{H}$ defined as $\mathrm{n} \times \mathrm{n}$ characteristic matrix $\mathrm{H}=\mathrm{D}-\mathrm{A}$, where $\mathrm{D}$ is the diagonal matrix of the degrees of $G$ and $A$ is the adjacency matrix of $\mathrm{G}, \mathrm{H}=\left[\mathrm{a}_{\mathrm{ij}}\right]$ defined as follows: (i) $a_{i j}=-1$ when $v_{i}$ and $v_{j}$ are adjacent and $i \neq j$, (ii) $a_{i j}$ equals the degree of vertex $v_{i}$ if $\mathrm{i}=\mathrm{j}$ and (iii) $\mathrm{a}_{\mathrm{ij}}=0$ otherwise. All of co-factors of $H$ are equal to $\tau(G)$. There are other methods for calculating $\tau(\mathrm{G})$. Let $\mu_{1} \geq \mu_{1} \geq \ldots \geq \mu_{p}$ denote the eignvalues of $H$ matrix of a $p$ point graph. Then it is easily shown that $\mu_{\mathrm{p}}=0$. Furthermore, Kelmans and Chelnokov (1974) shown that, $\tau(\mathrm{G})=\frac{1}{\mathrm{p}} \prod_{\mathrm{k}=1}^{\mathrm{p}-1} \mu_{\mathrm{k}}$. The formula for the number of spanning trees in a d-regular graph $\mathrm{G}$ can be expressed as $\tau(\mathrm{G})=\frac{1}{\mathrm{p}} \prod_{\mathrm{k}=1}^{\mathrm{p}-1}\left(\mathrm{~d}-\mu_{\mathrm{k}}\right)$ where $\lambda_{0}=\lambda_{1}, \lambda_{2}, \ldots ., \lambda_{\mathrm{p}-1}$ are the eigenvalues of the corresponding adjacency matrix of the graph. However, for a few special families of graphs there exist simple formulas that make it much easier to calculate and determine the number of corresponding spanning trees especially when these numbers are very large. One of the first such result is due to Cayley (1889) who showed that complete graph on $n$ vertices, $K_{n}$ has $n^{n-2}$ spanning trees that he showed $\tau\left(\mathrm{K}_{\mathrm{n}}\right)=\mathrm{n}^{\mathrm{n}-2}, \mathrm{n} \geq 2$. Another result, $\tau\left(\mathrm{K}_{\mathrm{p}, \mathrm{q}}\right)=\mathrm{p}^{\mathrm{q}-1} \mathrm{q}^{\mathrm{p}-1}, \mathrm{p}, \mathrm{q} \geq 1$, where $\mathrm{K}_{\mathrm{p}, \mathrm{q}}$ is the complete bipartite graph with bipartite sets containing $\mathrm{P}$ and $\mathrm{q}$ vertices, respectively. It is well known, as in e.g.,

(Clark, 2003; Qiao and Chen, 2009). Another result is due to Guy (1970) who derived a formula for the wheel on $n+1$ vertices, $W_{n+1}$, which is formed from a cycle $C_{n}$ on $n$ vertices by adding a vertex adjacent to every vertex of $C_{n}$. In particular, he showed that $\tau\left(\mathrm{W}_{\mathrm{n}+1}\right)=\left(\frac{3+\sqrt{5}}{2}\right)^{\mathrm{n}}+\left(\frac{3-\sqrt{5}}{2}\right)^{\mathrm{n}}-2$, for $\mathrm{n} \geq 3$. Sedlacek (1970) also later derived a formula for the number of spanning trees in a Mobius ladder. The Mobius ladder $M_{n}$ is formed from cycle $C_{2 n}$ on $2 n$ vertices labeled $v_{1}$, 
$\mathrm{v}_{2}, \ldots, \mathrm{v}_{2 \mathrm{n}}$ by adding edge $\mathrm{v}_{\mathrm{i}} \mathrm{v}_{\mathrm{i}+\mathrm{n}}$ for every vertex $\mathrm{v}_{\mathrm{i}}$ where $i \leq n$. The number of spanning trees in $M_{n}$ is given by $\tau\left(M_{n}\right)=\frac{n}{2}\left[(2+\sqrt{3})^{n}+(2-\sqrt{3})^{n}+2\right]$ for $n \geq 2$. Another class of graphs for which an explicit formula has been derived is based on a prism (Boesch and Bogdanowicz, 1987; Boesch and Prodinger, 1986). Let the vertices of two disjoint and length cycles be labeled $v_{1}, v_{2}, \ldots v_{n}$ in one cycle and $w_{1}, w_{2}, \ldots w_{n}$ in the other. The prism $R_{n}$ is defined as the graph obtained by adding to these two cycles all edges of the form $\mathrm{v}_{\mathrm{i}}, \mathrm{w}_{\mathrm{i}}$. The number of spanning trees in $R_{n}$ is given by the following formula $\frac{\mathrm{n}}{2}\left[(2+\sqrt{3})^{\mathrm{n}}+(2-\sqrt{3})^{\mathrm{n}}-2\right]$.

Lemma 1.1: Temperley (1964):

$$
\tau(\mathrm{G})=\frac{1}{\mathrm{p}^{2}} \operatorname{det}(\mathrm{H}+\mathrm{J})
$$

where, $\mathrm{J}$ is the $\mathrm{p} \times \mathrm{p}$ matrix, where all elements are unity.

We can also deduce the following lemma.

Lemma 1.2: $\tau(\mathrm{G})=\frac{1}{\mathrm{p}^{2}} \operatorname{det}(\mathrm{pI}-\overline{\mathrm{D}}+\overline{\mathrm{A}})$ where $\overline{\mathrm{A}}, \overline{\mathrm{D}}$ are the adjacency and degree matrices of $\overline{\mathrm{G}}$, the complement of $\mathrm{G}$, respectively and $\mathrm{I}$ is the $\mathrm{p} \times \mathrm{p}$ unit matrix.

The advantage of these formulas in lemma1.1, lemma 1.2 is to express $\tau(\mathrm{G})$ directly as a determinant rather than in terms of cofactors as in Kirchhoff theorem or eigenvalues as in Kelmans and Chelnokov formula.

Proof lemma 1.2: Since $\tau(G)=\frac{1}{p^{2}} \operatorname{det}(H+J), H=D-A$ then $\mathrm{D}=\mathrm{H}+\mathrm{A}, \quad$ since $\quad \mathrm{D}+\overline{\mathrm{D}}=(\mathrm{p}-1) \mathrm{I}, \quad$ then $\mathrm{D}=(\mathrm{p}-1) \mathrm{I}-\overline{\mathrm{D}} . \quad$ Thus $\mathrm{H}+\mathrm{A}=(\mathrm{p}-1) \mathrm{I}-\overline{\mathrm{D}}, \quad$ since $\mathrm{J}-\mathrm{A}=\mathrm{I}+\overline{\mathrm{A}}$. By addition we get $\mathrm{H}+\mathrm{J}=\mathrm{pI}-\overline{\mathrm{D}}+\overline{\mathrm{A}}$ and therefore $\tau(\mathrm{G})=\frac{1}{\mathrm{p}^{2}} \operatorname{det}(\mathrm{pI}-\overline{\mathrm{D}}+\overline{\mathrm{A}})$.

In our computations we need some lemmas on determinants and some relations concerning Chebyshev polynomials. We begin from their definitions, Zhang et al. (2005).

Let $A_{n}(x)$ be $n \times n$ matrix such that:

$$
\mathrm{A}_{\mathrm{n}}(\mathrm{x})=\left(\begin{array}{ccccc}
2 \mathrm{x} & -1 & 0 & & \\
-1 & 2 \mathrm{x} & -1 & 0 & \\
0 & \ddots & \ddots & & \ddots \\
& \ddots & \ddots & \ddots & -1 \\
& & 0 & -1 & 2 \mathrm{x}
\end{array}\right)
$$

where all other elements are zeros.

Further we recall that the Chebyshev polynomials of the first kind are defined by Eq. 1:

$T_{n}(x)=\cos (n \arccos x)$

The Chebyshev polynomials of the second kind are defined by Eq. 2:

$\mathrm{U}_{\mathrm{n}-1}(\mathrm{x})=\frac{1}{\mathrm{n}} \frac{\mathrm{d}}{\mathrm{dx}} \mathrm{T}_{\mathrm{n}}(\mathrm{x})=\frac{\sin (\mathrm{n} \arccos \mathrm{x})}{\sin (\arccos \mathrm{x})}$

It is easily verified that Eq. 3:

$U_{n}(x)-2 x U_{n-1}(x)+U_{n-2}(x)=0$

It can then be shown from this recursion that by expanding $\operatorname{det} \mathrm{A}_{\mathrm{n}}$ (x) one gets Eq. 4:

$\mathrm{U}_{\mathrm{n}}(\mathrm{x})=\operatorname{det}\left(\mathrm{A}_{\mathrm{n}}(\mathrm{x})\right), \mathrm{n} \geq 1$

Furthermore by using standard methods for solving the recursion (3), one obtains the explicit formula Eq. 5:

$$
\mathrm{U}_{\mathrm{n}}(\mathrm{x})=\frac{1}{2 \sqrt{\mathrm{x}^{2}-1}}\left[\left(\mathrm{x}+\sqrt{\mathrm{x}^{2}-1}\right)^{\mathrm{n}+1}-\left(\mathrm{x}-\sqrt{\mathrm{x}^{2}-1}\right)^{\mathrm{n}+1}\right], \mathrm{n} \geq 1
$$

where the identity is true for all complex $\mathrm{x}$ (except at $x= \pm 1$ where the function can be taken as the limit).

Lemma 1.3: Let $B_{n}(x)$ be $n \times n$ matrix such that:

$$
\mathrm{B}_{\mathrm{n}}(\mathrm{x})=\left(\begin{array}{cccccc}
\mathrm{x} & -1 & 0 & & & \\
-1 & 1+\mathrm{x} & -1 & 0 & & \\
0 & -1 & 1+\mathrm{x} & -1 & \ddots & \\
& \ddots & \ddots & \ddots & \ddots & 0 \\
& & \ddots & -1 & 1+\mathrm{x} & -1 \\
& & & 0 & -1 & \mathrm{x}
\end{array}\right)
$$

Then one can obtain:

$$
\operatorname{det}\left(B_{n}(x)\right)=(x-1) U_{n-1}\left(\frac{1+x}{2}\right)
$$

Proof: Straightforward induction using properties of determinants and above mentioned definition.

Lemma 1.4: Let $C_{n}(x)$ be $n \times n$ matrix, $n \geq 3, x>2$ then: 


$$
\mathrm{C}_{\mathrm{n}}(\mathrm{x})=\left(\begin{array}{cccccc}
\mathrm{x} & 0 & 1 & & & \\
0 & \mathrm{x}+1 & 0 & \ddots & & \\
1 & 0 & \mathrm{x}+1 & 0 & \ddots & \\
& \ddots & \ddots & \ddots & \ddots & 1 \\
& & \ddots & \ddots & \mathrm{x}+1 & 0 \\
& & & 1 & 0 & \mathrm{x}
\end{array}\right)
$$

Then:

$$
\operatorname{det}\left(C_{n}(x)\right)=(n+x-2) U_{n-1}\left(\frac{x}{2}\right)
$$

Proof: Straightforward induction using properties of determinants, we have:

$$
\operatorname{det}\left(\mathrm{C}_{\mathrm{n}}(\mathrm{x})\right)=\frac{\mathrm{n}+\mathrm{x}-2}{\mathrm{x}-2} \operatorname{det}\left(\begin{array}{cccccc}
\mathrm{x}-1 & -1 & 0 & & & \\
-1 & \mathrm{x} & -1 & 0 & & \\
0 & -1 & \mathrm{x} & -1 & \ddots & \\
& \ddots & \ddots & \ddots & \ddots & 0 \\
& & \ddots & \ddots & \mathrm{x} & -1 \\
& & & 0 & -1 & \mathrm{x}-1
\end{array}\right)
$$

Using lemma1.3 yields:

$\operatorname{det}\left(C_{n}(x)\right)=\frac{n+x-2}{x-2}(x-2) U_{n-1}\left(\frac{x}{2}\right)=(n+x-2) U_{n-1}\left(\frac{x}{2}\right)$

Lemma 1.5: If $B=\left(\begin{array}{cc}A & I \\ I & A\end{array}\right)$. Then $\operatorname{det}(B)=\operatorname{det}(A-I)$. $\operatorname{det}(\mathrm{A}+\mathrm{I})$.

Proof: Using the fact that:

$$
\operatorname{det}\left(\begin{array}{ll}
A & B \\
C & D
\end{array}\right)=\left\{\begin{array}{l}
\operatorname{det}(A) \cdot \operatorname{det}\left(D-C A^{-1} B\right) \\
\operatorname{det}(D) \cdot \operatorname{det}\left(A-B D^{-1} C\right)
\end{array},\right.
$$

where, A, D are non singular, Marcus and Minc (1964).

We have:

$$
\begin{aligned}
\operatorname{det}\left(\begin{array}{cc}
A & I \\
I & A
\end{array}\right) & =\operatorname{det}(A) \cdot \operatorname{det}\left(A-A^{-1}\right) \\
& =\operatorname{det}\left(A^{2}-I\right) \\
& =\operatorname{det}(A-I) \cdot \operatorname{det}(A+I) .
\end{aligned}
$$

This formula gives some sort of symmetry in some matrices which facilitates our calculation of determinants.

Complexity of cocktail party graphs: The cocktail party graph of order n, also called the hyper octahedral graph is the graph consisting of two rows of paired nodes in which all nodes but the paired ones are connected with a graph edge. It is the graph complement of the ladder rung graph $L_{n}$ and the dual graph of the hypercube graph $\mathrm{Q}_{\mathrm{n}}$. Biggs (1993). See Fig. 1.

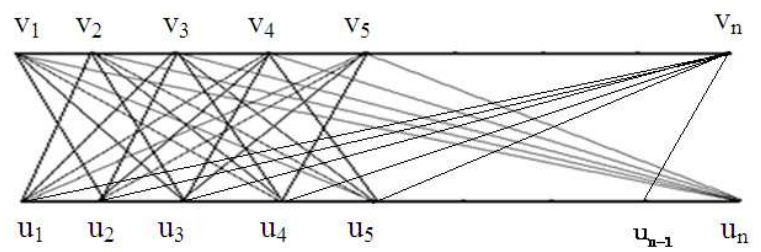

Fig. 1: Cocktail party graph with 2 n-vertices

Theorem 2.1: Let $\mathrm{G}$ be a cocktail party graph with $2 \mathrm{n}$ vertices, $n \geq 3$. Its complexity will be:

$$
\begin{aligned}
\tau(G) & =\frac{(n-1)}{n 2^{2 n} \sqrt{\left(n^{2}-4\right)\left((n+2)^{2}-4\right)}} \\
& \times\left[\left(n+\sqrt{n^{2}-4}\right)^{n}-\left(n-\sqrt{n^{2}-4}\right)^{n}\right] \\
& \times\left[\left((n+2)+\sqrt{(n+2)^{2}-4}\right)^{n}-\left((n+2)-\sqrt{(n+2)^{2}-4}\right)^{n}\right]
\end{aligned}
$$

Proof: Applying lemmas1.2 ,1.3,1.4 and1.5,we have:

$$
\begin{aligned}
& \tau(\mathrm{G})=\frac{1}{(2 \mathrm{n})^{2}} \operatorname{det}(2 \mathrm{n} \mathrm{I}-\overline{\mathrm{D}}+\overline{\mathrm{A}}) \\
& =\frac{1}{(2 \mathrm{n})^{2}} \operatorname{det} \\
& \left(\begin{array}{ccccc}
\mathrm{n}+1 & 0 & 1 & \ldots & 1
\end{array}\right. \\
& \begin{array}{cccccc}
0 & \mathrm{n}+2 & 0 & \ddots & \vdots & \\
1 & 0 & \ddots & \ddots & 1 & \text { I }
\end{array} \\
& \begin{array}{llll}
\ddots & \ddots & \mathrm{n}+2 & 0
\end{array} \\
& \begin{array}{lllll}
1 & \cdots & 1 & 0 & \mathrm{n}+1
\end{array} \\
& \begin{array}{lllll}
\mathrm{n}+1 & 0 & 1 & \cdots & 1
\end{array} \\
& \begin{array}{lllll}
0 & \mathrm{n}+2 & 0 & \ddots & \vdots
\end{array} \\
& \text { I } \\
& \begin{array}{llll}
1 & 0 & \ddots & 1
\end{array} \\
& \vdots \quad \ddots \ddots^{\mathrm{n}+2} \quad 0 \\
& \begin{array}{lllll}
1 & \cdots & 1 & 0 & \mathrm{n}+1
\end{array} \\
& =\frac{1}{(2 \mathrm{n})^{2}} \operatorname{det}\left(\begin{array}{ccccc}
\mathrm{n} & 0 & 1 & \cdots & 1 \\
0 & \mathrm{n}+1 & 0 & \ddots & \vdots \\
1 & 0 & \ddots & \ddots & 1 \\
\vdots & \ddots & \ddots & \mathrm{n}+1 & 0 \\
1 & \cdots & 1 & 0 & \mathrm{n}
\end{array}\right)
\end{aligned}
$$


Am. J. Applied Sci., 9 (2): 202-207, 2012

$$
\begin{aligned}
& \times \operatorname{det}\left(\begin{array}{ccccc}
\mathrm{n}+2 & 0 & 1 & \cdots & 1 \\
0 & \mathrm{n}+3 & 0 & \ddots & \vdots \\
1 & 0 & \ddots & \ddots & 1 \\
\vdots & \ddots & \ddots & \mathrm{n}+3 & 0 \\
1 & \cdots & 1 & 0 & \mathrm{n}+2
\end{array}\right) \\
& =\frac{1}{(2 n)^{2}} \frac{2 \mathrm{n}-2}{\mathrm{n}-2} \operatorname{det}\left(\begin{array}{ccccc}
\mathrm{n}-1 & -1 & 0 & \cdots & 0 \\
-1 & \mathrm{n} & -1 & \ddots & \vdots \\
0 & -1 & \ddots & \ddots & 0 \\
\vdots & \ddots & \ddots & \mathrm{n} & -1 \\
0 & \cdots & 0 & -1 & \mathrm{n}-1
\end{array}\right) \\
& \times 2 \operatorname{det}\left(\begin{array}{ccccc}
\mathrm{n}+1 & -1 & 0 & \cdots & 0 \\
-1 & \mathrm{n}+2 & -1 & \ddots & \vdots \\
0 & -1 & \ddots & \ddots & 0 \\
\vdots & \ddots & \ddots & \mathrm{n}+2 & -1 \\
0 & \cdots & 0 & -1 & \mathrm{n}+1
\end{array}\right) \\
& =\frac{1}{(2 n)^{2}} \frac{2 n-2}{n-2}\left[(n-2) U_{n-1}\left(\frac{n}{2}\right)\right] \times\left[2 n U_{n-1}\left(\frac{n+2}{2}\right)\right] \\
& =\frac{1}{(2 \mathrm{n})^{2}} \times \frac{2(\mathrm{n}-1)}{\mathrm{n}-2} \times(\mathrm{n}-2) \frac{1}{2^{\mathrm{n}} \sqrt{\mathrm{n}^{2}-4}} \\
& {\left[\left(\mathrm{n}+\sqrt{\mathrm{n}^{2}-4}\right)^{\mathrm{n}}-\left(\mathrm{n}-\sqrt{\mathrm{n}^{2}-4}\right)^{\mathrm{n}}\right]} \\
& \times 2 \mathrm{n} \frac{1}{2^{\mathrm{n}} \sqrt{(\mathrm{n}+2)^{2}+4^{2}}}[((\mathrm{n}+2)+ \\
& \left.\left.\sqrt{(\mathrm{n}-2)^{2}-4}\right)^{\mathrm{n}}-\left((\mathrm{n}+2)-\sqrt{(\mathrm{n}+2)^{2}-4}\right)^{\mathrm{n}}\right] \\
& =\frac{(\mathrm{n}-1)}{\mathrm{n} 2 \sqrt{2 \mathrm{n}} \sqrt{\left(\mathrm{n}^{2}-4\right)\left((\mathrm{n}+2)^{2}-4\right)}} \\
& x\left[\left(\mathrm{n}+\sqrt{\mathrm{n}^{2}-4}\right)^{\mathrm{n}}-\left(\mathrm{n}-\sqrt{\mathrm{n}^{2}-4}\right)^{\mathrm{n}}\right] \\
& x\left[\left((\mathrm{n}+2)+\sqrt{(\mathrm{n}-2)^{2}-4}\right)^{\mathrm{n}}-\left((\mathrm{n}+2)-\sqrt{(\mathrm{n}+2)^{2}-4}\right)^{\mathrm{n}}\right]
\end{aligned}
$$

Complexity of crown graph: A crown graph on $2 \mathrm{n}$ vertices is an undirected graph with two sets of vertices $u_{i}$ and $v_{i}$ and with an edge from $u_{i}$ to $v_{j}$ whenever $i \neq j$. The crown graph can be viewed as a complete bipartite graph from which the edges of a perfect matching have been removed, Biggs (1993) Fig. 2.

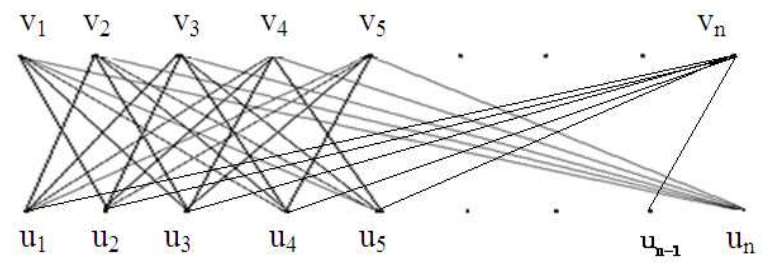

Fig. 2: Corwn graph with 2n-vertices

Lemma 2.2:Let $D_{n}(x)$ be $n \times n$ matrix such that:

$$
\mathrm{D}_{\mathrm{n}}(\mathrm{x})=\left(\begin{array}{cccccc}
\mathrm{x} & 1 & 1 & \cdots & \cdots & 1 \\
1 & \mathrm{x} & 1 & \ddots & & \vdots \\
1 & \ddots & \ddots & 1 & \ddots & \vdots \\
\vdots & \ddots & \ddots & \ddots & \ddots & 1 \\
\vdots & & \ddots & \ddots & \mathrm{x} & 1 \\
1 & \cdots & \cdots & 1 & 1 & \mathrm{x}
\end{array}\right)
$$

Then we obtain:

$$
\operatorname{det}\left(D_{n}\right)=(x+n-1)(x-1)^{n-1}
$$

Proof: From the definition of the circulant determinants, we have:

$$
\begin{aligned}
\operatorname{det}\left(D_{n}(x)\right) & =\operatorname{det}\left(\begin{array}{cccccc}
x & 1 & 1 & \cdots & \cdots & 1 \\
1 & x & 1 & \ddots & & \vdots \\
1 & \ddots & \ddots & 1 & \ddots & \vdots \\
\vdots & \ddots & \ddots & \ddots & \ddots & 1 \\
\vdots & & \ddots & \ddots & x & 1 \\
1 & \cdots & \cdots & 1 & 1 & x
\end{array}\right) \\
& =\prod_{j=1}^{n}\left(x+\omega_{j}+\omega_{j}^{2}+\omega_{j}^{3}+\ldots \ldots \ldots . .+\omega_{j}^{n-1}\right) \\
& =(x+1+1+\ldots \ldots .+1) \times \\
\prod_{j=1, \omega, \neq 1}^{n}(x+\underbrace{\left.\omega_{j}+\omega_{j}^{2}+\omega_{j}^{3}+\ldots \ldots \ldots .+\omega_{j}^{n-1}\right)}_{=-1} & =(x+n-1) \times(x-1)^{n-1} .
\end{aligned}
$$

Theorem 2.3: Let $\mathrm{G}$ be a crown graph with $2 \mathrm{n}$ vertices. Then the number of spanning trees of $G$ is:

$$
\tau(\mathrm{G})=\mathrm{n}^{\mathrm{n}-2}(\mathrm{n}-1)(\mathrm{n}-2)^{\mathrm{n}-1}
$$

Proof: Applying lemmas1.2 and 1.5 we have:

$$
\begin{gathered}
\tau(\mathrm{G})=\frac{1}{(2 \mathrm{n})^{2}} \operatorname{det}(2 \mathrm{n} \mathrm{I}-\overline{\mathrm{D}}+\overline{\mathrm{A}}) \\
\tau(\mathrm{G})=\frac{1}{(2 \mathrm{n})^{2}}\left(\begin{array}{cccccccc}
\mathrm{n} & 1 & \cdots & 1 & & & & \\
1 & \ddots & & \vdots & & & \mathrm{I} & \\
& & \ddots & 1 & & & & \\
1 & \cdots & 1 & \mathrm{n} & & & & \\
& & & & \mathrm{n} & 1 & \cdots & 1 \\
& & & 1 & \ddots & & \vdots \\
\mathrm{I} & & & \vdots & & \ddots & 1 \\
& & & 1 & \cdots & 1 & \mathrm{n}
\end{array}\right) \\
\\
=\frac{1}{(2 \mathrm{n})^{2}} \operatorname{det}\left(\begin{array}{ccccccc}
\mathrm{n}+1 & 1 & 1 & \cdots & & 1 \\
1 & \mathrm{n}+1 & 1 & \ddots & & \vdots \\
1 & 1 & \ddots & \ddots & 1 \\
\vdots & & \ddots & \ddots & \mathrm{n}+1 & 1 \\
1 & \cdots & 1 & 1 & \mathrm{n}+1
\end{array}\right)
\end{gathered}
$$




$$
\times \operatorname{det}\left(\begin{array}{ccccc}
\mathrm{n}-1 & 1 & 1 & \cdots & 1 \\
1 & \mathrm{n}-1 & 1 & \ddots & \vdots \\
1 & 1 & \ddots & \ddots & 1 \\
\vdots & \ddots & \ddots & \mathrm{n}-1 & 1 \\
1 & \cdots & 1 & 1 & \mathrm{n}-1
\end{array}\right)
$$

Applying lemma 2.2 with $\mathrm{x}=\mathrm{n}+1$ and $\mathrm{n}-1$ respectively, we have:

$$
\begin{aligned}
\tau(\mathrm{G}) & =\frac{1}{(2 \mathrm{n})^{2}} \times 2 \mathrm{n}^{\mathrm{n}} \times 2(\mathrm{n}-1)(\mathrm{n}-2)^{\mathrm{n}-1} \\
& =\mathrm{n}^{\mathrm{n}-2}(\mathrm{n}-1)(\mathrm{n}-2)^{\mathrm{n}-1}
\end{aligned}
$$

Complexity of open cocktail party graphs: The open cocktail party graph of order $\mathrm{n}$, is the graph consisting of cocktail party graph by deleting n-1 edges from one of their rows Fig. 3.

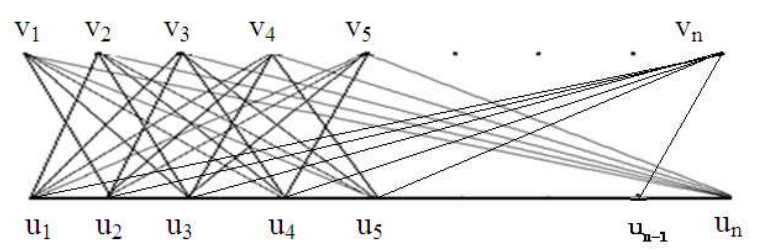

Fig. 3: Open cocktail party graph with 2 n-vertices

Lemma 2.4: If $A$ and $B$ are symmetric matrices and $\mathrm{C}=\left(\begin{array}{cc}\mathrm{A} & \mathrm{I} \\ \mathrm{I} & \mathrm{B}\end{array}\right)$.

Then:

$$
\operatorname{det}(\mathrm{C})=\operatorname{det}(\mathrm{AB}-\mathrm{I})=\operatorname{det}(\mathrm{BA}-\mathrm{I})
$$

Proof: Applying the fact that used in lemma 1.5.

Theorem 2.5: Let $\mathrm{G}$ be an open cocktail party graph with $2 \mathrm{n}$ vertices, $\mathrm{n} \geq 3$. Its complexity will be:

$$
\begin{aligned}
& \tau(G)=\frac{(n-1)^{n}}{n} U_{n-1}\left(\frac{n^{2}-2}{2(n-1)}\right) \\
& =\frac{n-1}{n \times 2 n \sqrt{n^{4}-8 n^{2}+8 n}} \times\left[\left(n^{2}-2+\sqrt{n^{4}-8 n^{2}+8 n}\right)^{n}-\right. \\
& \left.\quad\left(n^{2}-2-\sqrt{n^{4}-8 n^{2}+8 n}\right)^{n}\right]
\end{aligned}
$$

Proof: Applying lemmas (1.2), (1.4) and (2.4) we have:

$$
\tau(\mathrm{G})=\frac{1}{(2 \mathrm{n})^{2}} \operatorname{det}(2 \mathrm{n} \mathrm{I}-\overline{\mathrm{D}}+\overline{\mathrm{A}})
$$

$$
\begin{aligned}
& =\frac{1}{(2 \mathrm{n})^{2}} \operatorname{det}\left(\begin{array}{cccccccccc}
\mathrm{n} & 1 & 1 & \cdots & 1 & & & & & \\
1 & \mathrm{n} & 1 & \ddots & \vdots & & & & & \\
1 & 1 & \ddots & \ddots & 1 & & & \mathrm{I} & & \\
\vdots & \ddots & \ddots & \mathrm{n} & 1 & & & & & \\
1 & \cdots & 1 & 1 & \mathrm{n} & & & & & \\
& & & & & \mathrm{n}+1 & 0 & 1 & \cdots & 1 \\
& & & & 0 & \mathrm{n}+2 & 0 & \ddots & \vdots \\
& & \mathrm{I} & & & 1 & 0 & \ddots & & 1 \\
& & & & \vdots & \ddots & \ddots & \mathrm{n}+2 & 0 \\
& & & & & 1 & \cdots & 1 & 0 & \mathrm{n}+1
\end{array}\right) \\
& =\frac{1}{(2 n)^{2}} \operatorname{det}\left(\begin{array}{cccccc}
\left(\mathrm{n}^{2}+\right. & 2 \mathrm{n}-1 & 3 \mathrm{n}-2 & \ldots & \ldots & 3 \mathrm{n}-2 \\
2 \mathrm{n}-3) & & & & & \\
2 \mathrm{n}-1 & \left(\mathrm{n}^{2}+\right. & 2 \mathrm{n}-1 & 3 \mathrm{n}-2 & \ldots & \vdots \\
3 \mathrm{n}-2 & 2 \mathrm{n}-1 & \ddots & 2 \mathrm{n}-1 & 3 \mathrm{n}-2 & \vdots \\
\vdots & \ddots & \ddots & \ddots & \ddots & 3 \mathrm{n}-2 \\
\vdots & \ldots & \ddots & \ddots & \left(\mathrm{n}^{2}+\right. & 2 \mathrm{n}-1 \\
3 \mathrm{n}-2 & \ldots & \ldots & 3 \mathrm{n}-2 & 2 \mathrm{n}-1 & \left(\mathrm{n}^{2}+\right. \\
& & & & & 2 \mathrm{n}-3)
\end{array}\right) \\
& =\frac{(n-1)^{n}}{(2 n)^{2}} \operatorname{det}\left(\begin{array}{cccccc}
n+3 & \frac{2 n-1}{n-1} & \frac{3 n-2}{n-1} & \ldots & \ldots & \frac{3 n-2}{n-1} \\
\frac{2 n-1}{n-1} & n+4 & \frac{2 n-1}{n-1} & \frac{3 n-2}{n-1} & \ldots & \vdots \\
\frac{3 n-2}{n-1} & \frac{2 n-1}{n-1} & \ddots & \frac{2 n-1}{n-1} & \frac{3 n-2}{n-1} & \vdots \\
\vdots & \ddots & \ddots & \ddots & \ddots & \frac{3 n-2}{n-1} \\
\vdots & \ldots & \ddots & \ddots & n+4 & \frac{2 n-1}{n-1} \\
\frac{3 n-2}{n-1} & \cdots & \ldots & \frac{3 n-2}{n-1} & \frac{2 n-1}{n-1} & n+3
\end{array}\right)
\end{aligned}
$$

Straight forward induction by using properties of determinants.

We obtain:

$$
\begin{gathered}
\tau(G)=\frac{(n-1)^{n+1}}{(2 n-3) n^{2}} \times \operatorname{det} \\
\left(\begin{array}{cccccc}
\frac{n^{2}-2}{n-1} & 0 & 1 & \cdots & \cdots & 1 \\
0 & \left.\frac{n^{2}-2}{n-1}+1\right) & 0 & 1 & \cdots & \vdots \\
1 & 0 & \ddots & 0 & 1 & \vdots \\
\vdots & \ddots & \ddots & \ddots & \ddots & 1 \\
\vdots & \cdots & \ddots & \ddots & \left.\frac{n^{2}-2}{n-1}+1\right) & 0 \\
1 & \cdots & \cdots & 1 & 0 & \frac{n^{2}-2}{n-1}
\end{array}\right)
\end{gathered}
$$




$$
\begin{aligned}
& =\frac{(n-1)^{n+1}}{(2 n-3) n^{2}} \times \frac{2 n-3}{n-2} \times \text { det } \\
& \left(\begin{array}{cccccc}
\left(\frac{n^{2}-2}{n-1}-1\right) & -1 & 0 & \cdots & \cdots & 0 \\
-1 & \frac{n^{2}-2}{n-1} & -1 & 0 & \cdots & \vdots \\
0 & -1 & \ddots & -1 & 0 & \vdots \\
\vdots & \ddots & \ddots & \ddots & \ddots & 0 \\
\vdots & \cdots & \ddots & \ddots & \frac{n^{2}-2}{n-1} & -1 \\
0 & \cdots & \cdots & 0 & -1 & \left(\frac{n^{2}-2}{n-1}-1\right)
\end{array}\right) \\
& =\frac{(n-1)^{n+1}}{(n-2) n^{2}} \times\left(\frac{n^{2}-2}{n-1}-2\right) \times U_{n-1}\left(\frac{n^{2}-2}{2(n-1)}\right) \\
& =\frac{(n-1)^{n}}{n} \times U_{n-1}\left(\frac{n^{2}-2}{2(n-1)}\right) \\
& =\frac{n}{n \times 2^{n} \sqrt{n^{4}-8 n^{2}+8 n}}\left[\left(n^{2}-2+\sqrt{n^{4}-8 n^{2}+8 n}\right)^{n}\right. \\
& \left.-\left(n^{2}-2-\sqrt{n^{4}-8 n^{2}+8 n}\right)^{n}\right]
\end{aligned}
$$

\section{ACKNOWLEDGMENT}

The author is deeply indebted to the team of work at deanship of scientific research at Taibah university, for their continuous helps and the encouragement me to finalize this work. This research work was supported by a grant No. (625/1431) from the deanship of the scientific research at Taibah university, Al-Madinah Al-Munawwarah, K.S.A.

\section{REFERENCES}

Biggs, N., 1993. Algebraic Graph Theory. 2nd Edn., Cambridge University Press, Cambridge, ISBN: 0521458978, pp: 205.
Boesch, F.T. and H. Prodinger, 1986. Spanning tree formulas and chebyshev polynomials. Graphs Combinatorics, 2: 191-200. DOI: 10.1007/BF01788093

Boesch, F.T. and Z.R. Bogdanowicz, 1987. The number of spanning trees in a Prism. Int. J. Comput. Math., 21: 229-243. DOI: 10.1080/00207168708803568

Cayley, G.A., 1889. A theorm on trees. Quart. J. Math., 23: $276-378$.

Clark, L., 2003. On the enumeration of multipartite spanning trees of the complete graph. Bull. ICA, 38: 50-60.

Guy, R.K., 1970. Combinatorial Structures and their Applications. 1st Edn., Gordon and Breach, New York, pp: 508.

Kelmans, A.K. and V.M. Chelnokov, 1974 A certain polynomial of a graph and graphs with an extremal number of trees. J. Comb. Theory, 16: 197-214. DOI: 10.1016/0095-8956(74)90065-3

Kirchhoff, 1847. Uber die Auflosung der Gleichungen, auf welche man beider Untersuchung der Linearen Verteilung galvanischer Strme gefhrt wird. Ann. Phys. Chem., 72: 497-508.

Marcus, M. and H. Minc, 1964. A Survey of Matrix Theory and Matrix Inequalities. 1st Edn., Prindle, Weber and Schmidt, Boston, pp: 180.

Qiao, N.S. and B. Chen, 2009. The number of spanning trees and Chains of graphs. J. Applied Math. ENotes, 9: 10-16.

Sedlacek, J., 1970. Mathematics (Geometry and Graph theory) (Chech), University of Karlova.

Temperley, H.N.V., 1964. On the mutual cancellation of cluster integrals in Mayer's fugacity series. Proc. Phys. Soc., 83: 3-16. DOI: 10.1088/03701328/83/1/302

Zhang, Y., X Yong and M.J. Golin, 2005. Chebyshev polynomials and spanning tree formulas for circulant and related graphs. Discrete Math., 298: 334-364. DOI: 10.1016/j.disc.2004.10.025 